prominence to the statement that mice are not merely rendered immune by the blood of tetanus-immune rabbits, and that the protection of mice is brought about not merely when blood or serum of immune animals is injected into their peritoneal cavity immediately after infection, and before they have contracted tetanus; even when several extremities have already become tetanic, and when, according to previous experience, they might be expected to die in a few. hours if not treated-even then a cure can be effected with great certainty, and, indeed, so rapidly that within a few days no trace of disease is observable.

The possibility of a cure in the case of diseases with a quite acute course can therefore no longer be denied.

\title{
ON EXTRA-UTERINE PREGNANCY IN THE CAT.
}

By D. J. Hamilton, M.B., Professor of Pathology, Aberdeen University.

QUITE apart from the practical issues bound up with it, the subject of extra-uterine pregnancy considered from the purely biological point of view is one of great interest. The case which I am about to describe occurred in a cat, and is worth recording from extra-uterine pregnancy being a somewhat rare condition in the lower animals, as well as from the unique attachments which the progeny had contracted with neighbouring parts.

It is asserted that there are three situations in which primarily the embryo or embryos may be found: Firstly, occupying a Fallopian tube ; secondly, within the ovary itself; and, thirdly, growing from the peritoneum. The great majority of extra-uterine pregnancies, in Man at least, are acknowledged on all hands to be tubal in their origin. The foetus grows within the tube until about the end of the second or the beginning of the third month. It then bursts forth in one of two directions, either into the peritoneum or into the loose cellular tissue between the layers of the broad ligament.

In the case of the foetus escaping into the peritoneal sac, which is the commoner of the two occurrences, the parent dies sooner or later if unrelieved, and usually from abdominal hæmorrhage. The foetus, however, on rupturing into the abdomen need not immediately perish, nor need the condition prove at once fatal to the mother. On the contrary, the embryo may continue to grow in the peritoneal cavity until full time is reached, the placenta remaining adherent to the Fallopian tube, and the umbilical cord escaping through the rupture in its wall. 'The lacerated edges of the wound cicatrise, so that if examined some time after the accident has happened the cord is found to issue through an aperture with a regular clean cut margin ( $\mathrm{Sec}$ Chiari's case, Zeitschrift fur Heilkunde, vol. viii., i 887, p. 127).

Although the tube usually ruptures, yet it has been alleged that in some instances it goes on stretching up to the full term of gestation. A case recorded by Chiari (loc. cit., p. I28) appeared to have been of this nature. The child was fully nourished and was found lying in a sac supposed to be the distended Fallopian tube. A layer of muscular fibre lined the sac, said to be that of the tube in a state of 
hypertrophy. It is quite possible, however, that an extra-uterine pregnancy, originally tubal, might rupture into the broad ligament, become encysted, and, as affrmed by Tait, receive a covering. of muscular tissue from that membrane. The distended broad ligament might thus be mistaken for a Fallopian tube.

Where the rupture occurs into the broad ligament, the case may. ultimately prove fatal or not to the parent according to circumstances. Thus a lethal termination may be avoided by the foetus dying at an early period of development. It becomes encysted; and absorbed, or may be discharged piecemeal through an abscess. Sometimes the foetus after being encysted calcifies, and is thus converted into a lithopacdion. Such a foetus may remain in the tissues of the mother for a matter of thirty to forty years. A common accident, when the foetus ruptures into the broad ligament, is hæmorrhage from separation of the placenta. The blood escapes between the two layers of the ligament, and is a fertile source of one variety of pelvic hæmatocele.

Many explanations have been offered to account for these tubal pregnancies. It is generally stated that in the normal course of events the human spermatozooid meets the ovum somewhere in the Fallopian tube. Some say the union occurs close by the uterus; others that it takes place at the fimbriated end; while again it is asserted that the meeting point is about the middle of the tube. The last of these views has met with most acceptance. Tait, on the other hand (Ectopic Pregnancy and Peliic Hematocele, 1888), holds that impregnation is entirely uterine, that the lash of the cilia in the tube is towards the uterus, and that this is the natural safe-guard against tubal impregnation. When the tube suffers from any injury whereby the movements of its cilia are interfered with, as in catarrh, there is a. liability from the absence of this safe-guard to the spermatozooids passing up the tube and impregnating the ovum before its descent is completed. Another explanation afforded by Spiegelberg (Text-Book of Midwifery, Eng. Transl., N. Syd. Soc., I 887, p. 420) is that strictures of the tube, as from old adhesions, hinder the ovum from getting down but may not prevent the spermatozooid piercing upwards.

- The second alleged variety of extra-uterine pregnancy I have mentioned is where the ovary itself becomes the seat of impregnation. Much doubted as the possibility of this occurrence is by competent authorities, there are now at least thirteen (Spiegelberg) records of autopsies where an ovarian pregnancy is held to have been revealed. The ovum is said to be retained in the Graafian follicle at the time of rupture, and to become impregnated by the spermatozooids escaping from the end of the Fallopian tube. There is, further, the possibility that the fimbriated extremity of the tube may be adherent over a ripe Graafian vesicle and that ovarian impregnation may thus be favoured, it being granted that the spermatozooids have been able to make their way thus far up the tube. Such a series of events, however, seems conjectural and is certainly not likely often to happen. A fallacy connected with the interpretation of the appearances in cases of broad ligament foetation, namely, that the ovary is often stretched over and seems to be the source of the tumour, may have gone far to swell the records of this accident. Even granting that the above thirteen cases were all genuine instances of ovarian pregnancy, this form still 
remains, admittedly, the rarest of all varieties of extra-uterine fœtation.

A third contingency, however, may come to pass, namely, that the ovum after impregnation falls into the abdominal cavity. Tait (loc. cit., p. I 3) denies the possibility of this mishap ${ }^{1}$ on the grounds that the ovum would at once be destroyed by the digestive action of the abdomen. The abdomen, as Leopold's researches (Archive fiir Gynaekologie, vol. xviii., I 88 I, p. 53) have shown, has an extraordinary power of digesting the soft tissues of the dead foetus. Whether it has the same effect upon the freshly impregnated living ovum may be questioned, and indeed cases such as this $I$ am about to record seem to traverse any conclusion in the affirmative.

The cat from which I derived the beautiful specimen now in my possession was given to me by Professor Stirling some years ago. He found on killing the animal that the abdomen was occupied by several tumour masses, and judging that it was more a pathological subject than one suited for physiological purposes he sent it over to my laboratory. Being busy at the time, and not recognising the nature of the tumours from the fact of their being so bound up with the abdominal organs, I gave directions to have the viscera cut out and placed in a preservative fluid. To my astonishment on coming to examine the parts some time afterwards, I found that each tumour was a well developed kitten lying in the peritoneal sac. I had the whole preparation carefully set up, and as it now stands the description of it is as follows :-

There are four fully developed kittens all contained along with their adjuncta within the peritoneal cavity. Three of these are amassed in a somewhat pyramidal conglomerate measuring $7 \times 6$ ctm., and arising from the matting together of the parts concerned by dense fibrous adhesions. The kittens lie close to the middle of the body of the uterus, and are rolled up in a portion of the great omentum which is closely stretched over the sac in which each is contained. Running through the centre and so forming the axis of the tumour-like structure are the uterus and lower part of the intestine. The uterine horns had unfortunately been cut off close to their peripheral extremities, but sufficient of them remains to show that they are free from anything in the shape of a foetus-indeed, so far as one can judge, they seem to be in their virgin state. The body of the uterus is firmly clasped by the tumour mass, and its channel appears to have become impervious, apparently from the pressure of surrounding parts. Each of the three kittens involved is contained in a single membrane, rough internally but quite smooth externally. The exact manner of attachment of the placentæ cannot be distinctly traced owing to the fusion of the component elements.

The fourth foetus is entirely detached from the tumour formed by the other three. It has been growing from the lower edge of the great omentum, and appears to be the longest, measuring as it does between $\mathrm{I} 2$ and $\mathrm{I} 3 \mathrm{ctm}$. from the tip of the nose to the root of the tail. It is coiled up and like the others is enclosed within a single membrane, rough and granular internally but glabrous on the exterior. The membrane is so tough that it can be readily handled without

J Mr Bland Sutton informs me that he also cannot admit this as a source of extra-uterine pregnancy. 
injury. The placenta is situated upon the interior or rather seems to form part of this membrane. The area occupied by it is somewhat crescentic and from end to end measures from $2 \frac{1}{2}$ to $3 \mathrm{ctm}$. From it comes off what looks like a bunch of fibrous cords, but on closer inspection each of these proves to be an umbilical vessel. Nearer the fotus they are twisted into a rope-like texture, which makes a couple of coils round the right hind limb immediately above the foot. They afterwards penetrate the abdomen in the usual situation, the abdominal wall being perfectly closed.

The omental basis to which the placenta is attached is constituted by a few of the islands of fat naturally present within the membrane. The loose omental trabecular tissue for some distance above this has become adherent to and bound up with these particles of fat so as to constitute a solid stump to the free extremity of which the placenta is united.

There is an entire absence of anything like recent peritonitis, and the only evidence of its having existed formerly is in the localised adhesions whereby the three first described foetus are lashed together and to the body of the uterus. The nutrition of the mother like that of the offspring was excellent.

From the foregoing it will be gathered that, while the uterus was free from any signs of utero-gestation, four kittens had formed a placental attachment to the peritoneal surface and had derived their maternal sustenance from the peritoneal blood-vessels. There is this peculiarity in the case as compared with most of those previously recorded, namely, that the embryos were not all attached to the peritoneum in the neighbourhood of the uterus and tubes, but that one of them was growing from the great omentum and was quite free even from any inflammatory adhesion to the internal organs of generation. As a rule, when the placenta has attached itself to the peritoneum, it is that portion of the membrane adjacent to the Fallopian tubes which is selected. It has been supposed that on account of this part of the peritoneal sac being practically continuous with the mucous membrane of the tube, it bears some analogy to it. It represents, moreover, the portion of the pleuro-peritoneal sac which gives birth to the essential elements of the ovary, and hence has been held to be essentially genital in its character, and has been looked upon as the only part of the peritoneum which may vicariously take on the function of a uterus. The above case disproves this theory, for although certainly three of the foetus were growing from the pelvic peritoneum, the remaining fotus was far removed from it. It seems more rational to suppose that the usual attachment to the pelvic peritoneum is to be accounted for by the ova falling into it more readily than into other subdivisions of the sac. At the same time it seems one of the most extraordinary phenomena in nature that the wall of a serous cavity should thus assume functions entirely foreign to it.

Tait and others have alleged that these extra-uterine pregnancies with a peritoneal attachment of the placenta have originally been tubal, that the tube has burst, that the escaped placenta has wandered outwards, and that, slug-like, the placenta has fastened itself to some part of the peritoneum. I cannot bring myself to believe in such an occurrence, more especially when we find as in the instance just related 
that not only one foetus has escaped, but that four of them have each a separate point of connection with the peritoneal membrane. The only explanation admissible under these circumstances is that the fecundated ova fell either from the ovary directly or from the end of the tube into the abdominal cavity, and took root upon the parts of the peritoneum with which they came in contact. If it be the case that they were rejected from the tube, it comes to be a question how this arose. Quite possibly it may have been through a suddenly reversed peristalsis.

\title{
DISSEMINATED NECROSIS OF THE LIVER OF THE OX AND SHEEPx
}

\author{
By J. M'Fadyean, M.B., B.Sc., F.R.S.E., Royal Veterinary \\ College, Edinburgh.
}

UNDER the above title I intend to describe what is, if not a rare, at least an uncommon, disease of the liver of the domestic ruminants. It is not to my knowledge described in any of the text-booksEnglish or foreign-and I know of only one short reference to it, which I shall here quote at length $:^{1}-$

"In the liver of cattle there sometimes occur nodules of various dimensions which on section are dry and yellowish-brown, and allow the original acinous marking to be still recognised. These nodules are necrotic pieces of liver. Sections of these nodules were prepared after hardening in alcohol, and stained with alkaline methylene-blue solution. With slight magnification these showed the following picture. The dead liver tissue was completely colourless, stained parts were first visible at the limit between the dead and the living substance of the liver; these were wavy lines which were directed at right angles to the dead tissue. At some places these bundles penetrated in the form of spears into the tissue. With high magnification a structure could no longer be recognised in the unstained tissue; only in the marginal part of the nodule badly stained long bacilli were visible here and there. Farther outwards they became more and more numerous, and this was succeeded by long wavy streaks and dense bundles of the same, which penetrated into the sound liver tissue. When portions of these nodules were inoculated into the anterior chamber of the eye of rabbits, the eye became filled with a mass of interwoven bacilli and became caseous; the bacilli also grew in the direction of the optic nerve as far as the brain. When the ear was inoculated with portions of the nodules it became swollen and caseous, and the cavity of the tympanum became filled with the bacillary growth. In mice inoculated subcutaneously on the back, the bacilli grew through the muscles into the abdominal cavity. These were perfectly classical pictures of perforation of a tissue determined by growth of bacilli."

My own observations regarding this lesion date from the year 1886 , when I received from Mr Winter of Limerick, at that time a student at the veterinary college, a portion of the liver of a cow which had been under treatment by Mr Donald of Wigton. This piece of liver

\footnotetext{
I Schuetz, Der Streptococcus der Druse der Pferde. Arehir. f. Wissen. und Prakt. Thierheilkunde, $18 s 8$.
} 